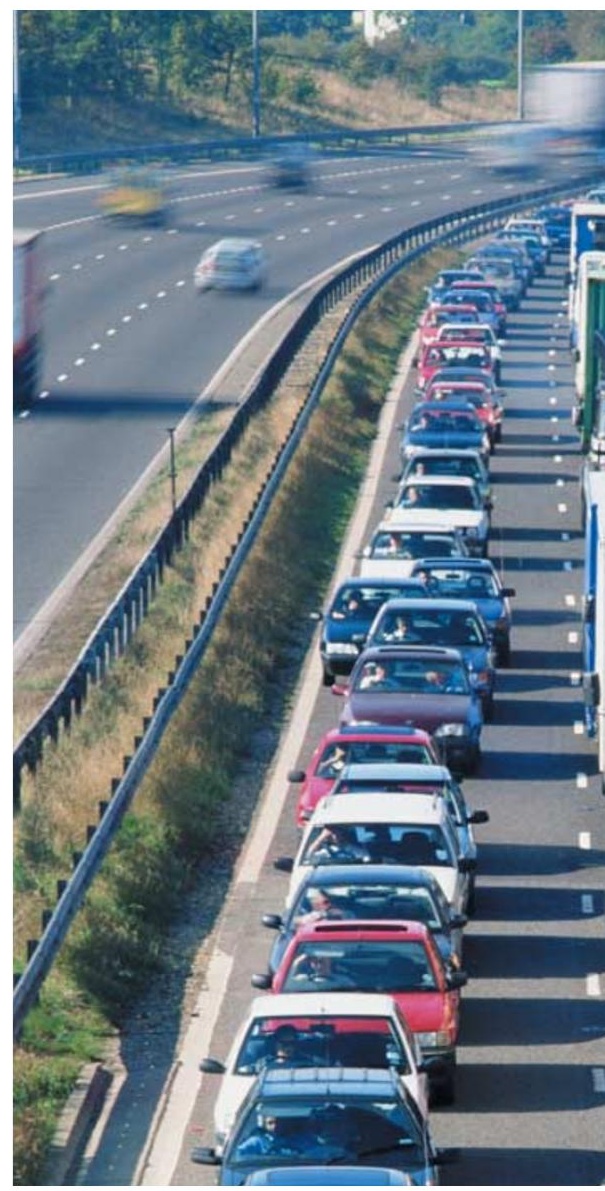

Neurons depend heavily on efficient intracellular transport, perhaps more than any other type of cell. Two papers in Neuron provide evidence that proteins with expanded glutamine repeats, such as huntingtin, might interfere with axonal transport, and that this might contribute to the development of neurodegeneration in polyglutamine disorders such as Huntington's disease.

In the first study, Gunawardena et al. looked at axonal transport in Drosophila. Flies in which the expression of huntingtin had been reduced by tissue-specific RNAi showed accumulation of organelles within larval nerves, which is typical of a deficit in axonal transport. In addition, reduction of huntingtin in the eye caused neurodegeneration in the eyes of adult flies, similar to that caused by overexpression of mutant huntingtin. These results indicate that normal huntingtin might be involved in axonal transport. But similar

deficits were produced by expression of a fragment of huntingtin containing the expanded polyglutamine region, pointing towards a toxic gain of function.

Further investigation revealed that the levels of soluble motor proteins in neurons from flies expressing polyglutamine proteins were reduced. Huntingtin is known to interact with huntingtin-associated protein 1 (HAP1), which in turn associates with a component of the axonal transport system, and the authors suggest that the mutant protein might sequester motor proteins. The resulting shortage of motor proteins in the cell could cause the deficits in axonal transport.

By targeting expression of polyglutamine proteins to either the nucleus or the cytoplasm of cells, Gunawardena et al. showed that the axonal transport phenotype depended on cytoplasmic expression of the proteins. When expression was restricted to the nucleus, though, it triggered apoptosis in neurons. The authors propose that mutant huntingtin has two distinct pathogenic mechanisms, one that disrupts transport and another that triggers apoptosis, and that they might act together in Huntington's disease.
The Times (UK) points out (7 October, 2003), "MRI is especially useful for examining the brain and spinal cord. Nearly all brain disorders produce differences in water content, which show up on the MRI scan."

Of course, for many neuroscientists the ultimate development of MRI is functional MRI, which allows the indirect visualization of changes in neural activity in the human brain. Talking to The Times, Lauterbur said, "There is one very interesting area in the observation of processes going on in the brain, to determine parts of the brain that respond to various thoughts and perceptions." The techniques involved in these studies are still being improved, but the fundamentals go back to the seminal work of Mansfield, Lauterbur and others in the 1970s.

\title{
GLIA
}

\section{Tight unions}

There is much more to the nodes of Ranvier than simply being axonal segments devoid of myelin. The architecture of the region of contact between oligodendrocytes or Schwann cells and the nerve fibre is exquisitely complex, and two recent papers provide new insight on the molecular interactions between the two cell types.

The myelin sheath has been divided in several regions paranode, juxtaparanode and internode - that have different structural and molecular signatures. The studies of Poliak et al. and Traka et al. focus on the juxtaparanode, asking what molecular interactions are necessary for its proper assembly. Previous studies had shown that $\mathrm{K}^{+}$ channels are enriched at the axonal membrane of the juxtaparanode, where they seem to interact indirectly with the protein Caspr2. The new studies provide evidence that the adhesion molecule Tag1, which is expressed by axon and glia at the juxtaparanode, is crucial for the localization of $\mathrm{K}^{+}$channels and Caspr2.

Poliak et al. generated mice lacking Caspr2 and found that $\mathrm{K}^{+}$ channels and Tag1 were no longer abundant in the juxtaparanodes of the peripheral and central nervous systems. To study these interactions in more detail, they generated mice lacking Tag1 and found a similar phenotype: $\mathrm{K}^{+}$channels and Caspr2 were missing from the juxtaparanode. A series of biochemical experiments helped them to obtain evidence for the direct interaction between Tag1 and Caspr2, and to show that they only interact when they form part of the same membrane and not between cells. Traka et al. did not generate Caspr2-null mice, but working with a Tag1 knockout, they obtained similar findings: the abnormal localization of $\mathrm{K}^{+}$channels and Caspr2 in the mutant, and the interaction between Tag1 and Caspr2 in cis, but not in trans.

Both studies converge on the idea that Tag1 is crucial for the assembly of the juxtaparanode on two different fronts. First, owing to its association with Caspr2, Tag1 helps to define the molecular composition of the juxtaparanode. 


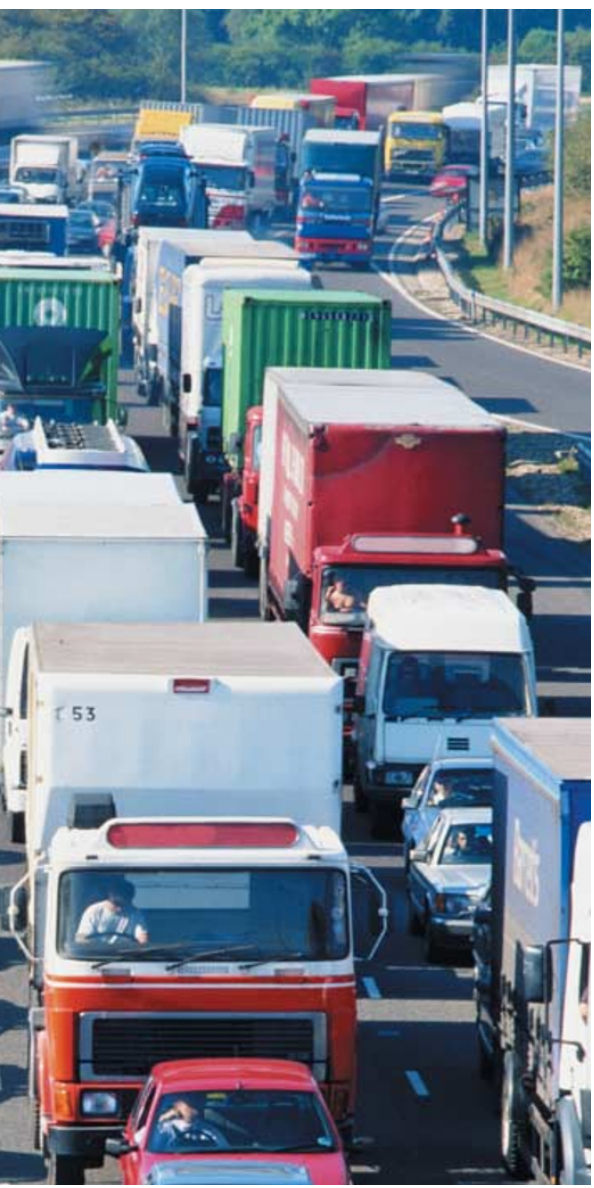

In the second study, Szebenyi et al. used isolated axoplasm from squid giant axons to investigate fast axonal transport. When the axoplasm was perfused with a mutant huntingtin fragment, both anterograde and retrograde fast axonal transport were slowed. Another polyglutamine protein, the androgen receptor (expansions in which are associated with spinobulbar muscular atrophy), had the same effect. The authors also found that the mutant androgen receptor inhibited neurite outgrowth in neuronal cell lines, further supporting the idea that the protein interfered with axonal transport.

In Huntington's disease and other polyglutamine disorders, the mutated proteins accumulate in both the nucleus and the cytoplasm. It is unclear which of these contributes to the pathology, or even whether aggregates are necessary for neurodegeneration. These studies add another piece to the puzzle, but we are clearly still a long way from a full understanding of how polyglutamine expansions cause neurodegeneration.

\section{(2) References and links} Rachel Jones ORIGINAL RESEARCH PAPERS Gunawardena, S. et al. Disruption of axonal transport by loss of huntingtin or expression of pathogenic polyQ mutants in Drosophila. Neuron 40, 25-40 (2003) Szebenyi, G. et al. Neuropathogenic forms of huntingtin and androgen receptor inhibit fast axonal transport. Neuron 40, 41-52 (2003) FURTHER READING Muqit, M. M. K. \& Feany, M. B. Modelling neurodegenerative disorders in Drosophila: a fruitful approach? Nature Rev. Neurosci. 3, 237-243 (2002)
Second, as this adhesion molecule forms homophilic interactions between opposing membranes (in this case, between axon and glia), Tag1 participates in maintaining the structural integrity of this region of the myelin sheath. Juan Carlos López

\section{(2) References and links} ORIGINAL RESEARCH PAPERS Poliak, S. et al. Juxtaparanodal clustering of Shaker-like K channels in myelinated axons depends on Caspr2 and TAG-1. J. Cell Biol. 162, 1149-1160 (2003) | Traka, M. et al. Association of TAG-1 with Caspr2 is essential for the molecular organization of juxtaparanodal regions of myelinated fibers. J. Cell Biol. 162 1161-1172 (2003)

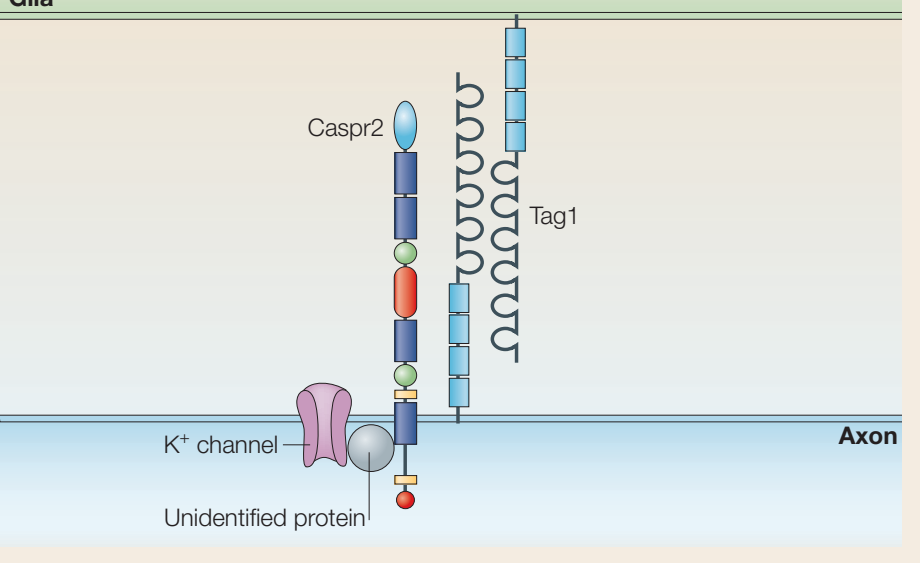

\section{IN BRIEF}

\section{ION CHANNELS}

Structural basis for modulation and agonist specificity of HCN pacemaker channels.

Zagotta, W. N. et al. Nature 425, 200-205 (2003)

HCN channels have pacemaking activity in heart and brain cells, are activated by hyperpolarization, and are modulated by cyclic nucleotides. On the basis of crystallographic and equilibrium sedimentation analyses of the carboxy-terminal domain of HCN2, Zagotta and colleagues define the mechanism of cyclic nucleotide specificity and identify a domain that mediates the tetramerization of this channel region. They also propose a possible mechanism for the allosteric modulation of gating. These results might be relevant to other cyclic nucleotidebinding channels.

\section{SENSORY SYSTEMS}

Multiple actions of systemic artemin in experimental neuropathy.

Gardell, R. L. et al. Nature Med. 5 October 2003 (doi: 10.1038/nm944)

Neuropathic pain - pain caused by functional disturbances of peripheral nerves - can be successfully treated with systemic artemin in rats, according to this study. Artemin is a member of the glial-derived neurotrophic factor family, and the peripheral expression of its receptor - GFR $\alpha 3$ - is restricted to nociceptive neurons. The authors found that artemin reversed several behavioural, morphological and biochemical correlates of experimental neuropathy with no obvious side effects, pointing to its possible therapeutic use.

\section{COGNITIVE NEUROSCIENCE}

Sounds and silence: an optical topography study of language recognition at birth.

Peña, M. et al. Proc. Natl Acad. Sci. USA 100, 11702-11705 (2003)

Newborn infants can organize the auditory world.

Winkler, I. et al. Proc. Natt Acad. Sci. USA 100, 11812-11815 (2003)

Our understanding of the cognitive abilities and organization of newborn infants suffers from difficulties in applying traditional techniques of study to babies. In these two papers, different techniques are applied to show that the responses of newborn infants to auditory stimuli are similar to those of adults. In the first, Peña et al. use optical topography — an optical technique for imaging changes in blood flow in the cortex below the scalp - to show that, even in babies that are just two or three days old, the left hemisphere responds preferentially to speech, but not to backward speech. In the second study, Winkler and colleagues use the mismatch negativity — an electroencephalogram signal that is a signature for the detection of an 'oddball' stimulus - to show that similarly young infants can also segregate concurrent streams of sound according to their source. As in adults, the segregation of the sounds depends on a difference in spatial pitch between the two streams. 